\title{
The Stratospheric THz Observatory (STO)
}

\author{
C. Walker ${ }^{1}$, C. Kulesa ${ }^{1}$, P. Bernasconi ${ }^{2}$, H. Eaton ${ }^{2}$, N. Rolander ${ }^{2}$, C. Groppi ${ }^{3}$, J. Kloosterman ${ }^{1}$, T.

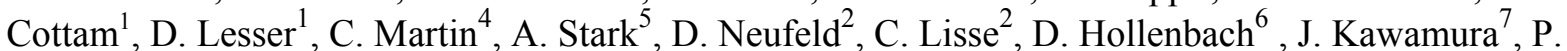 \\ Goldsmith $^{7}$, W. Langer ${ }^{7}$, H. Yorke ${ }^{7}$, J. Sterne ${ }^{7}$, A. Skalare ${ }^{7}$, I. Mehdi ${ }^{7}$, S. Weinreb ${ }^{8}$, J. Kooi ${ }^{8}$, J.

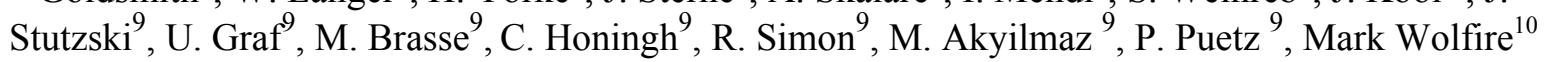 \\ ${ }^{1}$ Steward Observatory, University of Arizona, Tucson, AZ 85721 USA \\ ${ }^{2}$ Johns Hopkins Applied Physics Laboratory, Laurel, MD 20723 USA \\ ${ }^{3}$ School of Earth and Space Exploration, Arizona State University, Tempe, AZ 85287 USA \\ ${ }^{4}$ Oberlin College, Oberlin, OH 44074 USA. \\ ${ }^{5}$ Smithsonian Astrophysical Observatory, Cambridge, MA 02138 USA \\ ${ }^{6}$ SETI Institute, Mountain View, CA 94043 USA. \\ ${ }^{7}$ Jet Propulsion Laboratory, Pasadena, CA 91109 USA \\ ${ }^{8}$ California Institute of Technology, Pasadena, CA 91125 USA \\ ${ }^{9}$ University of Cologne, Cologne, D-50937 Germany \\ ${ }^{10}$ University of Maryland, College Park, MD 20740 USA \\ *Contact: cwalker@as.arizona.edu, +01-520-621-8783
}

\begin{abstract}
The Stratospheric TeraHertz Observatory (STO) is a NASA funded, Long Duration Balloon (LDB) experiment designed to address a key problem in modern astrophysics: understanding the Life Cycle of the Interstellar Medium (ISM). STO will survey a section of the Galactic plane in the dominant interstellar cooling line [C II] (1.9 THz) and the important star formation tracer [N II] $(1.46 \mathrm{THz})$ at $\sim 1$ arc minute angular resolution, sufficient to spatially resolve atomic, ionic and molecular clouds at $10 \mathrm{kpc}$. STO itself has three main components; 1) an $80 \mathrm{~cm}$ optical telescope, 2) a THz instrument package, and 3) a gondola [1]. Both the telescope and gondola have flown on previous experiments [2,3]. They have been reoptimized for the current mission. The science flight receiver package will contain four [CII] and four [NII] HEB mixers, coupled to a digital spectrometer. The first engineering test flight of STO was from Ft. Sumner, NM on October 15, 2009. The $\sim 30$ day science flight is scheduled for December 2011 .
\end{abstract}

Keywords: Suborbital, Balloon-borne, THz astronomy, Interstellar medium

\subsection{INTRODUCTION}

STO is a Long Duration Balloon (LDB) experiment designed to address a key problem in modern astrophysics: understanding the Life Cycle of the Interstellar Medium (ISM). During its upcoming science flight STO will survey a section of the Galactic plane in the dominant interstellar cooling line [C II] $(158 \mu \mathrm{m})$ and the important star formation tracer [N II] $(205 \mu \mathrm{m})$ at 1 arcminute angular resolution, sufficient to spatially resolve atomic, ionic and molecular clouds at $10 \mathrm{kpc}$. The goals for the survey are to:

[1] Determine the life cycle of Galactic interstellar gas.

[2] Study the creation and disruption of star-forming clouds in the Galaxy.

[3] Determine the parameters that affect the star formation rate in the galaxy.

[4] Provide templates for star formation and stellar/interstellar feedback in other galaxies.

Ground-based and Airborne Telescopes III, edited by Larry M. Stepp, Roberto Gilmozzi, Helen J. Hall Proc. of SPIE Vol. $7733,77330 \mathrm{~N} \cdot$ (C) 2010 SPIE · CCC code: 0277-786X/10/\$18 - doi: 10.1117/12.857765 
On Oct. 15, 2009 STO had its test flight from Ft. Sumner, NM. During its $\sim 12$ hours at float altitude $(\sim 126,000$ $\mathrm{ft}$.) key components of STO were tested to help ensure the system would meet the objectives of the upcoming science flight. STO consists of 3 major components; a gondola, an $80 \mathrm{~cm}$ telescope, and a THz heterodyne receiver system. The gondola and telescope have been refurbished from the successful Flare Genesis Experiment. The gondola was upgraded by APL to use 3 gyroscopes for inertial guidance and an optical tracker for absolute pointing. The telescope was light-weighted and its primary and secondary mirrors re-aluminized. A room temperature receiver system and a cryostat were constructed and flown on the test flight. A computercontrolled, sliding weight was added to dynamically compensate for cryogen evaporation during flight.

\subsection{TEST FLIGHT INSTRUMENT}

The STO flight from New Mexico was designed to provide a faithful test of the Antarctic gondola, telescope, and key elements of the science instrumentation.

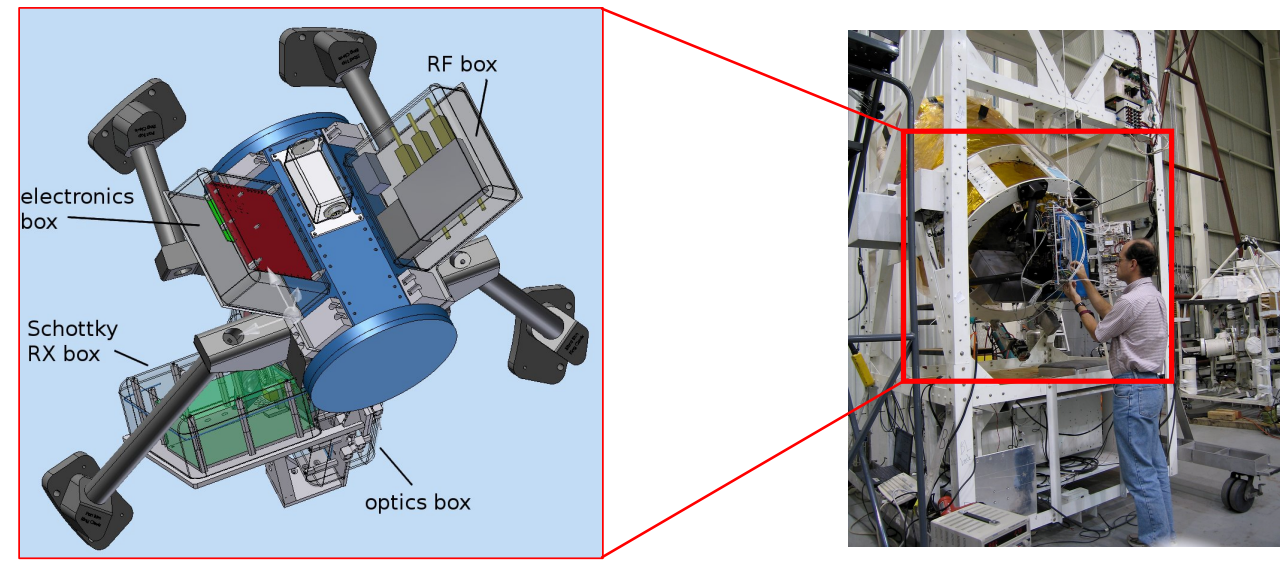

Figure 1: STO test-flight telescope and instrument package configuration, as conceptualized (left) and being integrated at Fort Sumner in early October, 2009 (right).

Antarctic ready versions of the telescope/gondola systems, instrument electronics, computing, control, and data storage systems were flown. To faithfully characterize the performance of the telescope drives and pointing system, the test flight instrument was designed with the same CG and weight as the full Antarctica flight instrument. The STO test flight carried a test cryostat and an un-cooled Schottky receiver. The receiver was used to point on the ${ }^{13} \mathrm{CO} \mathrm{J}=3-2$ line. The dewar was used to test the ability of a sliding weight to compensate for the loss of cryogens during flight.

Figure 1 shows the STO telescope and instrument package as it was configured for the test flight. The instrument flight system as deployed contained the following subsystems: 


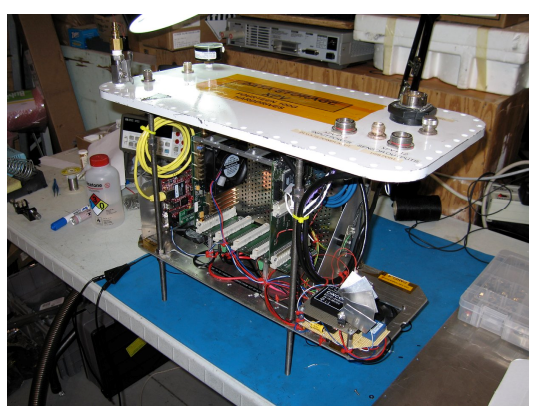

Figure 2: Pressure vessel containing the data acquisition and control computer, solid state storage, network router, and Omnisys FFT spectrometer.

- an optics box containing relay optics for the $330 \mathrm{GHz}$ receiver and actuators to direct the telescope beam into calibration loads.

- an electronics box containing the instrument control computer, bias electronics for the Schottky and future HEB receivers, housekeeping temperature monitors, analog multiplexers, and solid state relays for calibration actuators.

- a RF box containing the LO drive synthesizers, amplifiers for the HEB mixers, and a downconverter for the Schottky mixer receiver.

- a $330 \mathrm{GHz}$ Schottky mixer receiver from JPL, kept at ambient temperature and pressure.

- a $4 \mathrm{~K}$ dewar, the nitrogen and helium cryogenic vessels were pressure-regulated to approximately sea level pressure, to maintain a helium bath temperature of $4.2 \mathrm{~K}$.

- A pressure vessel containing the FFT spectrometer, data control computer, and Cisco router to isolate the instrument network traffic from the gondola. Figure 2 shows the pressure vessel contents just before they were sealed and installed onto the gondola mezzanine level.

Figure 3 depicts the subsystem contents in a block diagram.

End-to-end testing of the complete system was performed on the ground using the ambient temperature Schottky receiver. A $330 \mathrm{GHz}$ test tone was emitted by an outdoor transmitter through the telescope and instrument optics (Figure 4). 


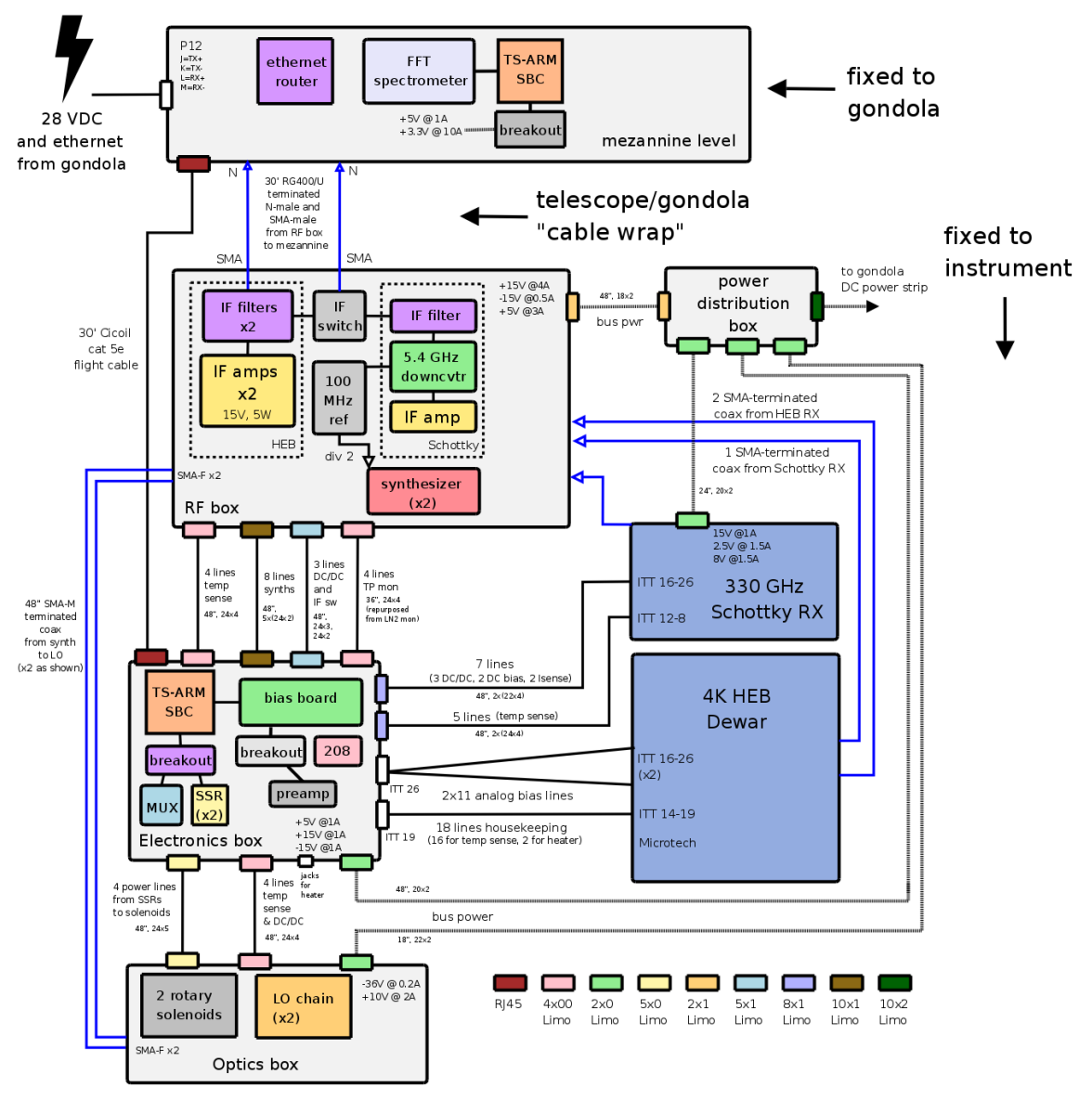

Figure 3: Detailed block diagram of the instrument control electronics and their location on the gondola.
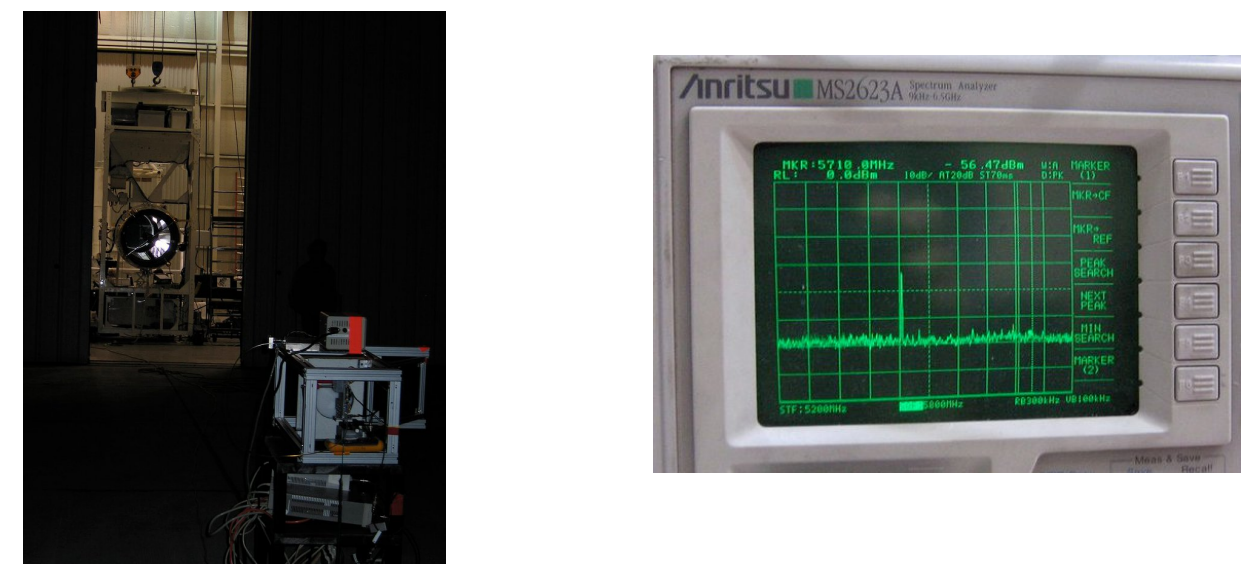

Figure 4: (Left) End-to-end testing of the entire STO instrument using the ambient temperature Schottky receiver and a $330 \mathrm{GHz}$ test transmitter placed $\sim 50 \mathrm{~m}$ away from the telescope. (Right) Spectrum analyzer view of the Schottky receiver IF, showing the test transmitter tone passing through all telescope and instrument optics and downconverted and amplified by the IF processor. This test represented the final "go" for launch. 


\subsection{TEST FLIGHT RESULTS}

STO was uneventfully launched at 10:03 AM MDT on 15 October, 2009 from the NASA facility at Fort Sumner, New Mexico under excellent launch conditions (Figure 5).
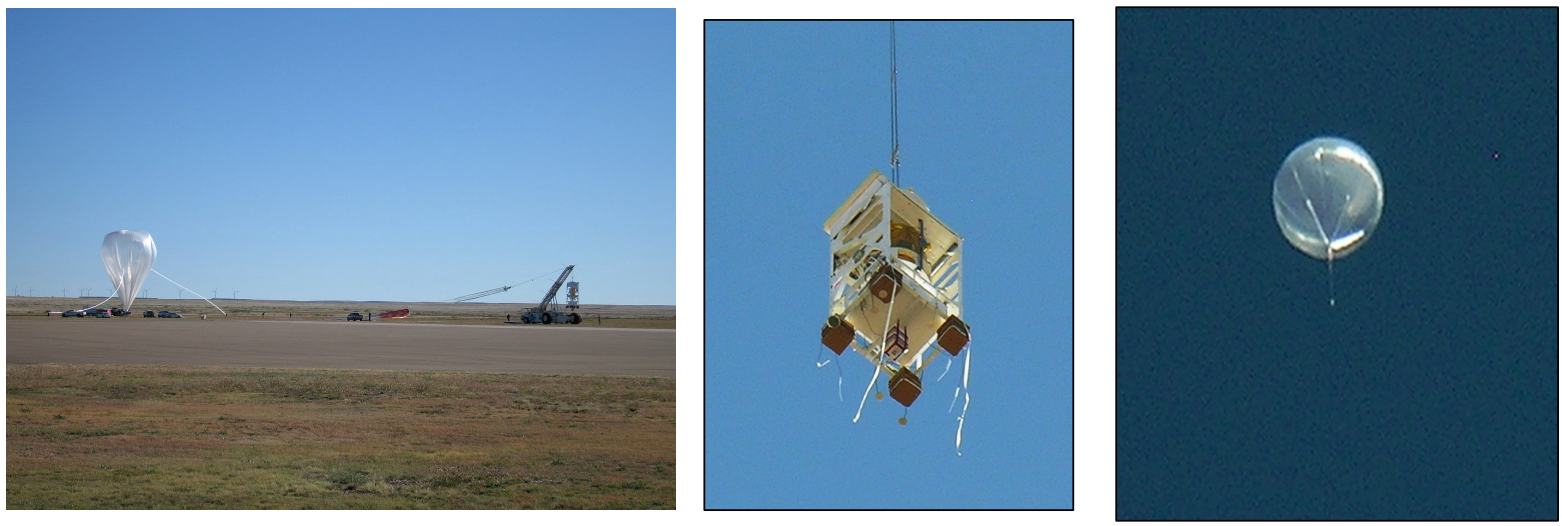

Figure 5: (Left) Balloon inflation is nearly complete at 9:50 AM on 15 October. (Center) STO launches at 10:03 AM, and reaches float altitude two hours later, hanging overhead (Right) for nearly the entire flight, and landing $<100$ miles away at 1:15 AM on 16 October.

The primary results from the test flight were:

1) The gyros performed well, allowing the telescope/gondola to track at the 5 arcsecond level (rms) after settling from a slew or a momentum transfer.

2) The star tracker demonstrated it can provide absolute pointing knowledge that meets mission requirements.

3) The sliding weight successfully controlled the telescope CG during all phases of flight.

4) The room temperature receiver, instrument control electronics, IF processor, and spectrometer performed well throughout the flight.

5) Control of the telescope, gondola, and instrument was maintained throughout the flight.

6) As an end-to-end test of the system, ${ }^{13} \mathrm{CO} \mathrm{J}=3-2$ spectrum of the Orion molecular cloud was obtained by STO at float altitude (see Figure 6). 


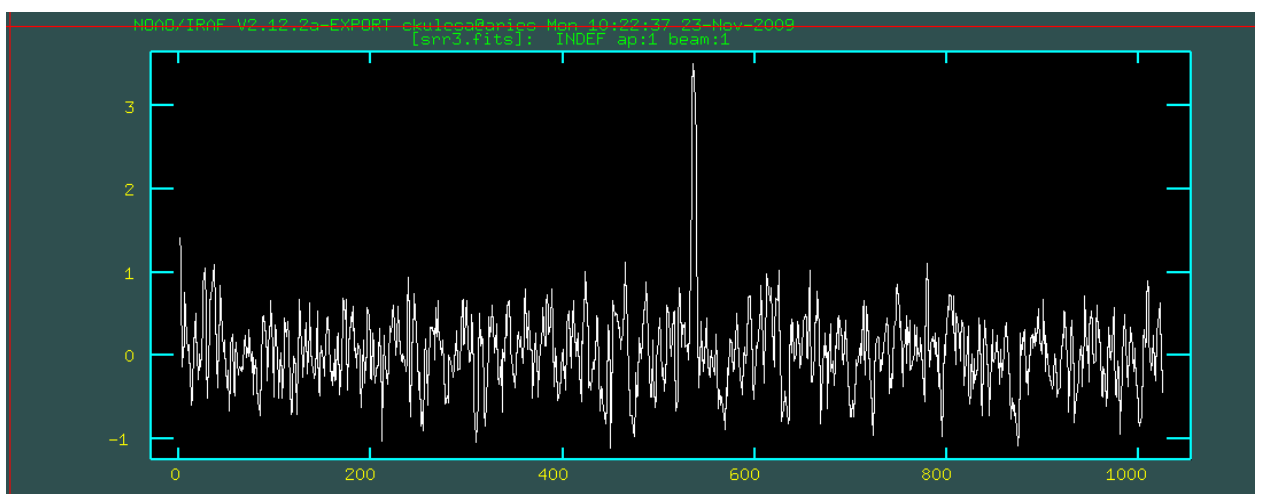

Figure 6: STO first-light spectral observation of the ${ }^{13} \mathrm{CO} \mathrm{J}=3-2$ line toward Orion taken from float altitude.

\subsection{PLANS FOR 2011 SCIENCE FLIGHT}

STO is scheduled to have its first LDB science flight from McMurdo, Antarctica in December 2011. For the science flight STO will employ a long ( $\sim 28$ day) hold time cryostat. A 3-D model of the planned science flight instrument configuration is shown in Figure 7. To achieve the goals of the science flight STO will fly two 4pixel arrays of heterodyne receivers operating at $1.9 \mathrm{THz}$ and $1.46 \mathrm{THz}$, the frequencies of the [CII] and [NII] lines respectively. Niobium superconducting hot electron mixers (similar to those used on Herschel - HiFi) provide state-of-the-art noise performance at $\mathrm{THz}$ frequencies. These mixers operate just below the transition temperature of niobium at $9 \mathrm{~K}$. Therefore, a key component of STO is a cryogenic system to maintain the mixers at this operating temperature for the duration of the flight. For the 2011 science flight, a liquid helium dewar will be used for this purpose. Figure 7a illustrates how the dewar will be mounted to the telescope.

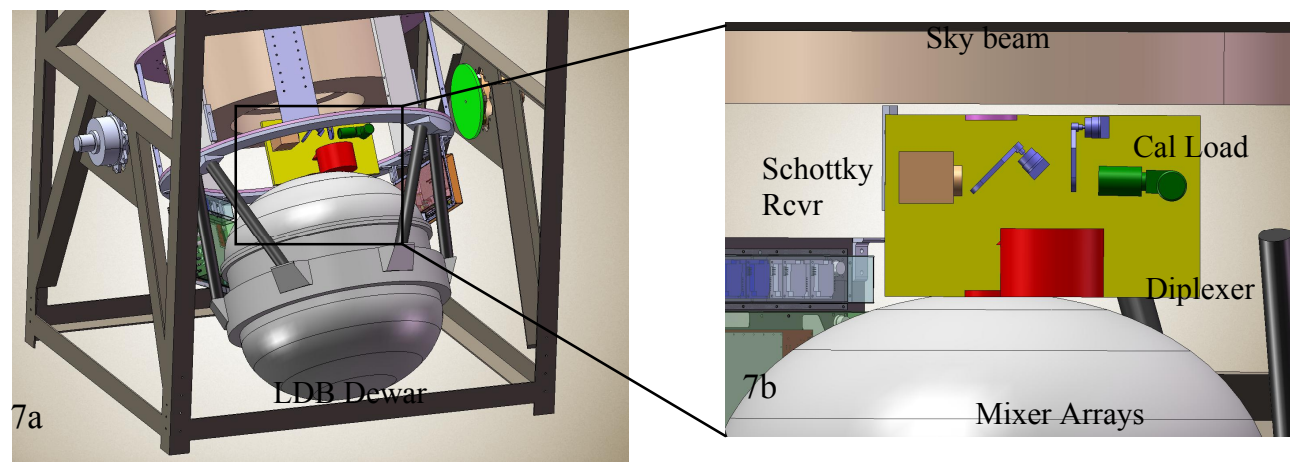

Figure 7: LDB dewar mounted to STO telescope. a) Dewar and optics box attached to telescope ring. b) Closeup of optics box containing relay optics, $330 \mathrm{GHz}$ Schottky receiver, flip mirrors, calibration load, and FabryPerot ring diplexer.

Between the telescope and the dewar is an optics box (see Figure 7b) containing flip mirrors for directing the $f / 17$ telescope beam to either a room temperature Schottky receiver, calibration loads, or a Fabry-Perot ring diplexer designed to efficiently inject the local oscillator (LO) beams into the telescope beam (Figure 8). The 
LO beams originate from two solid-state sources (not-shown; one at 1.9 and the other at $1.46 \mathrm{THz}$ ) whose output are divided into 4 equal power beams by a Fourier grating. From the diplexer, the combined telescope $+\mathrm{LO}$ beams enter the dewar through a $130 \mathrm{~mm}$ diameter window and continue to a wire grid located on the $4 \mathrm{~K}$ cold plate. The grid splits the beams into horizontal and vertical polarizations. The horizontal polarized components proceed to the $1.9 \mathrm{THz}$ mixer array and the vertical components to the $1.46 \mathrm{THz}$ mixer array. Offaxis mirrors convert the $\mathrm{f \#}$ of the telescope beam to match that expected by each mixer's feedhorn. As shown in the figure, each $2 \times 2$ mixer array is formed by bolting individual mixers to a common ring.

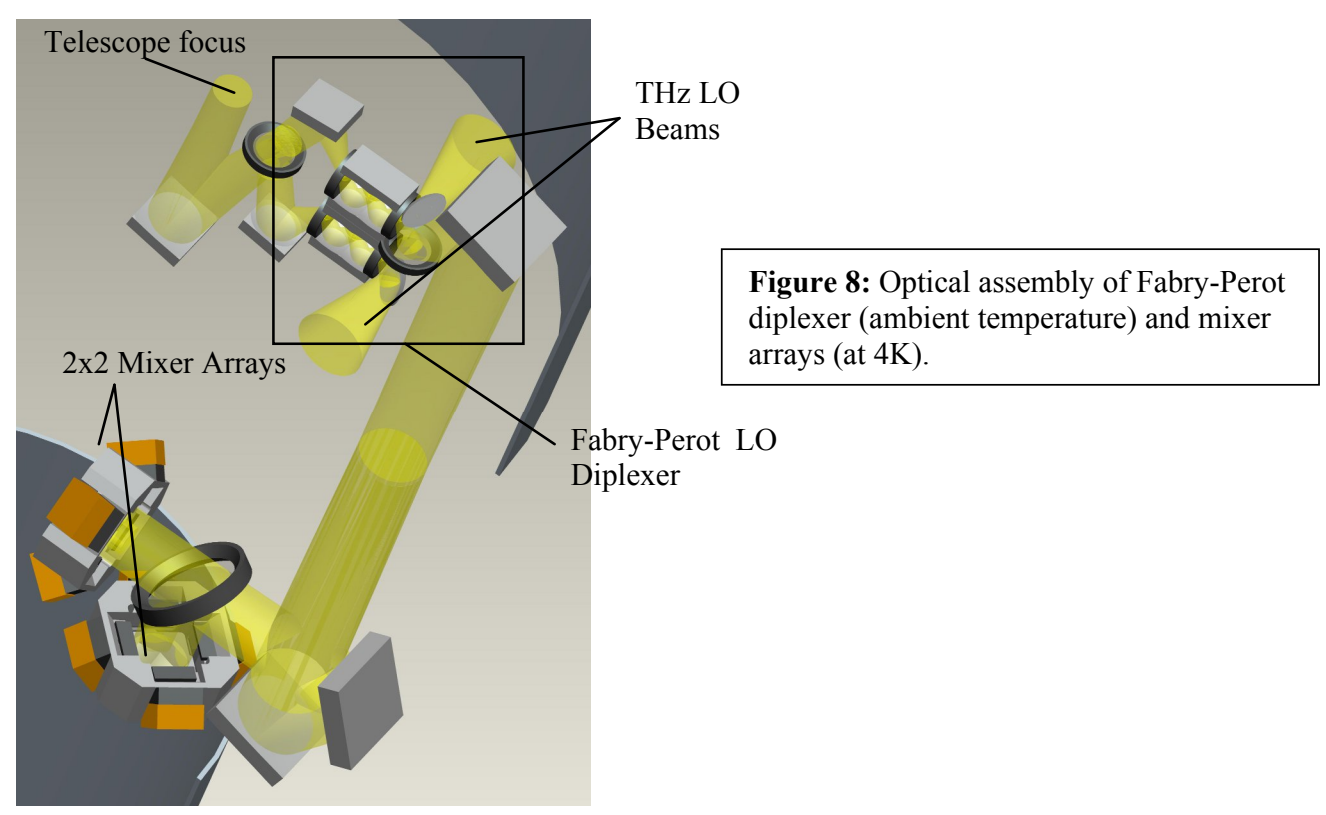

The hot electron bolometer (HEB) mixers for the [NII] arrays are being provided by JPL and the HEB mixers for the [CII] arrays by the University of Cologne. Waveguide technology is being used by both groups to meet the instrument noise and optical coupling requirements. A plot showing the characteristic performance of the mixers is shown in Figure 9. 


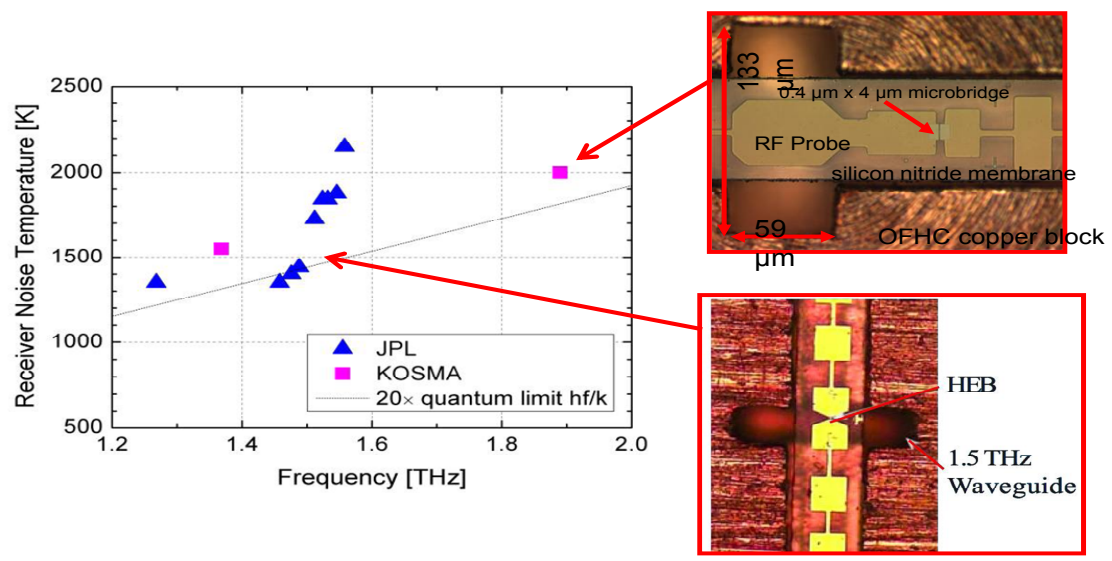

Figure 9: Performance and waveguide structures of JPL and Cologne mixers for STO

Each HEB device is DC biased to operate at the transition point between a normal conductor and a superconductor. The impending local oscillator signal acts to switch the HEB between the two states. In the process the telescope signal is also switched, with serves to multiply the telescope and LO signals together. The product of this multiplication contains both sum and difference frequencies between the two. The signal at the difference frequency is referred is the intermediate frequency (IF) signal. The IF signal for STO has a bandwidth of $1 \mathrm{GHz}$ and is centered at $1.5 \mathrm{GHz}$, appropriate for low noise operation of our HEBs. The IF output of each mixer is conveyed through coaxial cable to low-noise, low-power amplifiers operating at a physical temperature of $\sim 40 \mathrm{~K}$. These amplifiers (see Figure 10) were designed and fabricated by Co-I Weinreb at Caltech specifically for STO.
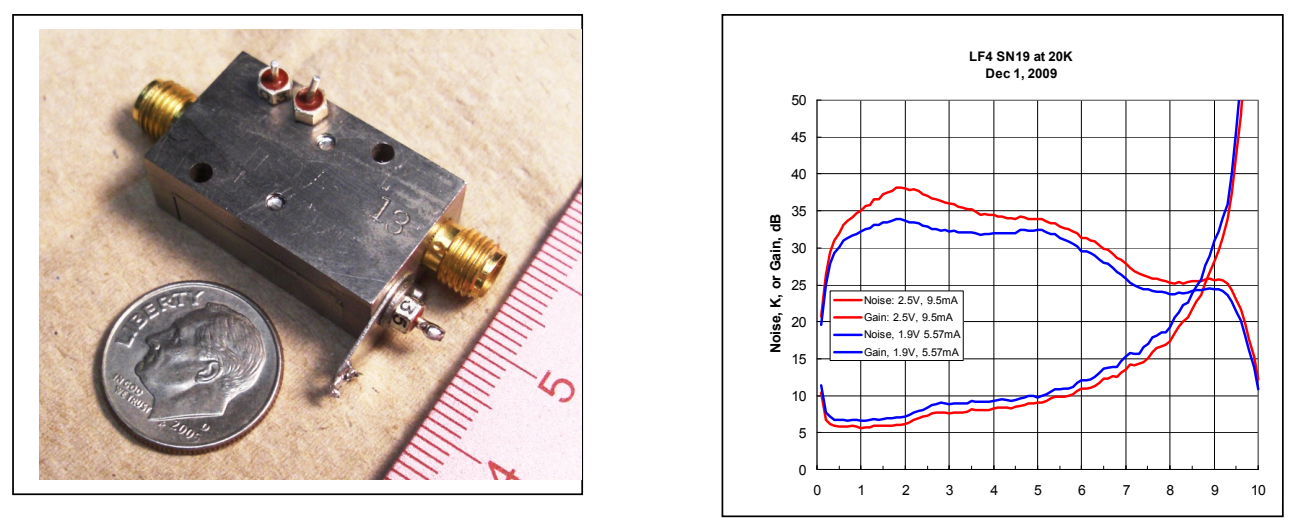

Figure 10: STO IF amplifiers (left). Performance curves (right).

After being amplified $\sim 30 \mathrm{~dB}$, the IF signals are brought out of the dewar via coaxial cable to the IF Processor bolted on the frame of the telescope (see Figure 11). The IF Processor Box further amplifies the signal from each HEB mixer and provides a DC signal proportional to each pixel's total power. An Electronics Box, also 
bolted to the telescope frame, contains a bias card and computer for the receiver frontend. From the IF processor eight coaxial cables (one for each focal plane mixer) carry the amplified IFs up to the pressurized vessel containing the spectrometer (see Figure 2) located at the mezzanine level of the gondola. The pressure vessel contains a single board computer and the digital FFT spectrometer that digitizes and performs FFTs on each of the eight, $1 \mathrm{GHz}$ wide, input signals. The resulting power spectra are stored in non-volatile flash memory and made available to the gondola computer via ethernet. The IF Processor, DC Electronics, and digital FFT spectrometers for the science flight are only slightly modified versions of what flew on the test flight.

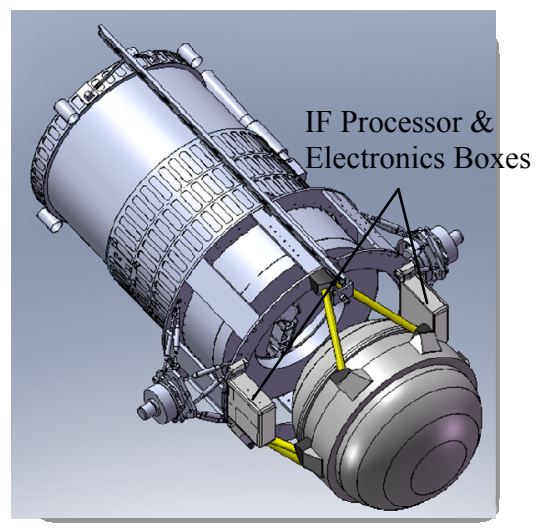

Figure 11: Location of IF Processor and Electronics Boxes on telescope assembly.

\section{REFERENCES}

[1] Walker, C. K., Kulesa, C. A., Groppi, C. E, Young,, E. T., McMahon, T., Bernasconi, P.,. Lisse, C., Neufeld, D, Hollenbach, D., Kawamura, J., Goldsmith, P., Langer, W.,. Yorke, H., Sterne, J., Skalare, A., Mehdi, I., Weinreb, S., Kooi, J., Stutzki, J., Graf, U., Honingh, N., Puetz, P., Martin, C., Wolfire, M., "The Stratospheric TeraHertz Observatory" in Proceedings of 19th International Symposium on Space Terahertz Technology, ed. Wolfgang Wild, Groningen, 28-30 April 2008, (https://www.sron.nl/files/LEA/ISSTT2008/Proceedings_ISSTT2008.pdf), p.28 (2000).

[2] Bernasconi, P.N., Rust, D. M., Eaton, H. A. C., Murphy, G. A. A., “A Balloon-borne Telescope for high resolution solar imaging and polarimetry", in Airborne Telescope Systems, Ed. By R. K. Melugin, and H. P. Roeser, SPIE Proceedings, 4014, 214 (2000).

[3] Bernasconi, P.N., Eaton, H. A. C., Foukal, P., and Rust, D. M., '“The Solar Bolometric Imager’, Adv. Space Res., 33, 1746 (2004). 